\title{
A Framework for Efficient Process Development Using Optimal Experimental Designs
}

\author{
Peter van de Ven • Sabina Bijlsma • Erik Gout • \\ Kees van der Voort Maarschalk • Uwe Thissen
}

Published online: 17 February 2011

(C) Springer Science+Business Media, LLC 2011

\begin{abstract}
Introduction The aim of this study was to develop and demonstrate a framework assuring efficient process development using fewer experiments than standard experimental designs.

Methods A novel optimality criterion for experimental designs (Iw criterion) is defined that leads to more efficient process development because: (a) prior knowledge is used in the experimental design to focus on optimal processing conditions and (b) a lean design is used which can dramatically reduce the
\end{abstract}

P. van de Ven $\cdot$ S. Bijlsma $\cdot$ U. Thissen

TNO (Dutch Organization for Applied Scientific Research), Business Unit Quality and Safety,

Zeist, The Netherlands

P. van de Ven

Department of Epidemiology and Biostatistics, VU University

Medical Center,

Amsterdam, The Netherlands

E. Gout

Abbott Healthcare Products, Oral Solid Dosage form

Development,

Weesp, The Netherlands

K. van der Voort Maarschalk

Department of Pharmaceutical Technology and Biopharmacy, University of Groningen,

Groningen, The Netherlands

K. van der Voort Maarschalk

Oral and Polymeric Products Development Department, MSD,

Oss, The Netherlands

U. Thissen $(\triangle)$

TNO Quality of Life,

P.O. Box 360, NL-3700 AJ, Zeist, The Netherlands

e-mail: Uwe.Thissen@tno.nl

URL: www.tno.nl number of experiments compared to standard designs. In this way, the criterion serves as a framework to connect a series of screening and optimization designs.

Results The philosophy behind the Iw criterion is explained including a detailed step-wise discussion how to apply it in practice. Moreover, its advantages were shown in an industrial process development case using a screening and an optimization design that were not explicitly connected. In this paper, a reduction of $21 \%$ of experiments could be obtained compared to the traditional approach using standard experimental designs and no framework.

Conclusions The Iw criterion is a valuable tool to increase accuracy and to speed up research that contain sets of experiments and where prior knowledge is already available or will be derived using screening designs.

Keywords Design of experiments (DOE) - Optimal design · I optimal · Quality by design · Process development

\section{Introduction}

The main objective of pharmaceutical process development is to design a manufacturing process that consistently yields products of good quality. Besides crucial, this is also a challenging task, as manufacturing processes typically consist of several unit operations in which a large number of (process and input) variables affect the quality of the final product.

Because of its expenses in time and money, there is a large pressure to perform process development as concise as possible. However, proper process development is also crucial to ensure high quality and cost-efficient manufacturing. One of the greatest challenges is to obtain quantitative information about interactions between (process and input) variables with reasonable effort. The 
importance of interactions between variable is clearly defined by the FDA's definition of the design space: "The multidimensional combination and interaction of input variables (e.g. material attributes) and process parameters that have been demonstrated to provide assurance of quality. Working within the design space is not considered as a change. Movement out of the design space is considered to be a change and would normally initiate a regulatory post approval change process. Design space is proposed by the applicant and is subject to regulatory assessment and approval" [1].

It is evident that always some number of experiments is needed to design the process (i.e., determine the design space by finding appropriate process settings). Furthermore, it is widely recognized that standard experimental designs, such as (fractional) factorial designs, orthogonal arrays, or PlackettBurman designs offer large benefits over change-oneparameter-at-a-time experiments. For a clear overview of the use of experimental designs in the pharmaceutical industry, we refer to $[2,3]$. Frequently, it is shown that experimental designs bear the advantage of requiring less experiments and providing more reliable results, specifically about interactions. However, pharmaceutical process development will benefit even more from well-constructed experimental designs if standard experimental designs (e.g., from textbooks or software) are replaced by approaches where results from one set of experiments are used to select the design of experimentation in a next stage: a multi-stage sequential design approach. The advantage of this approach using prior knowledge is twofold: (a) the total study is more focused on the latest insights of the most interesting parameter settings and (b) a statistical optimality criterion can be used to ensure the smallest possible number of experiments.

As an example, in the literature, Bayesian D-optimal designs have recently been proposed as a means to reduce the experimental effort in a pharmaceutical process development by using existing (i.e., prior) information in combination with an optimality criterion [4]. In these situations, optimal design theory chooses the settings for the factors in an experiment in such a way that the expected amount of information in the experiment is maximized. For the regular D-optimality criterion, maximum information is defined by maximizing the determinant of the variance-covariance matrix for the estimators of regression coefficients (and, equivalently, the volume of the confidence ellipsoid for these coefficients). Bayesian D-optimal designs are an extension to the regular D-optimal designs where information can be taken into account that is already available on the values of the regression coefficients. This prior information is incorporated in the design criterion by specification of a prior distribution for the parameters. The idea of using available information in choosing the experimental strategy can be of great benefit in the sequential experimental procedures used in pharmaceutical process development. The Bayesian Dcriterion, however, focuses on an accurate estimation of the unknown parameters instead of finding the design space, which is the essential reason of performing the experiments. Therefore, solely relying on the D-optimality criterion may result in a larger focus on combinations of parameter settings for which it is unlikely that the final product will meet all requirements. This will lead again to performing more experiments than strictly required.

In this paper, we present an alternative design criterion which specifically takes into account the latest insights on the best set of parameter settings: the weighted, multivariate I-criterion (Iw). The major benefit is that it focuses on the region for which it is expected that all requirements are met. The criterion is based on the standard I-criterion and minimizes a weighted average prediction variance over the experimental region. This novel and weighted criterion assures that the criterion gives more weight to design points that correspond to settings of the input and process parameters for which it is likely that a final product will meet the requirements (referred to a "preliminary design space" in this document with the likeliness judged on the basis of previous experiments). Typically, the designs that are optimal with respect to the weighted I-criterion cover the whole experimental region, but a larger proportion of the design points is located in and at the edges of the region where acceptance of the final product is deemed most likely. In contrast to the D-criterion, the I-criterion minimizes the error of the predictions, which corresponds to an increased reliability of the design space.

The goal of this paper is to explain the strategy of a multistage sequential design strategy for process optimization where a novel Iw criterion is used to assure optimal experiments (i.e., a minimal number of experiments with a maximal reliability). The benefits of this approach are illustrated by comparing its experimental load in an industrial case study which only used conventional designs that were not connected in a framework. Because of the mathematical basis of this new approach, its theoretical power and performance will not be affected compared to the traditional design.

\section{Materials and Methods}

\section{Criteria for Optimization}

A typical design approach consists of two steps: first a screening study is performed to identify the most important parameters and variables (perhaps preceded by a desk study), followed by a new designed more consecutive optimization experiment. In contrast, the approach proposed in this paper explicitly uses the results of the screening study to tune the optimization design. As an 
optimization design, a so-called optimal design is used. Optimal designs are experimental designs that are optimal with respect to a certain criterion. This criterion measures how well a design performs with respect to a predefined goal, such as the ability to estimate some unknown model parameters or the prediction of a response variable. Optimality criteria make it possible to compare different designs $[5,6]$.

The previously mentioned D-criterion is based on maximizing the information matrix which corresponds to minimizing the total variance of the estimators (i.e., the model parameters). In this study, the focus lies on finding the settings of process parameters that are optimal for the product quality. This means that a design is needed that is optimal with respect to the predicted values (model outcome) and not to the model parameters. The optimality criterion used in this study is based on the I-criterion which minimizes the average prediction variance by minimizing the mean squared prediction error. In this paper we propose an extension of the I-criterion: a multivariate and weighted version of the I-criterion.

\section{Adapted I-Optimality Criterion}

Consider a standard linear model that relates a (univariate) quality parameter vector $\mathrm{y}$ of random observations to a column vector $\beta$ of unknown parameters: $y=\mathbf{X} \beta+\varepsilon$, where $\mathbf{X}$ is an $n$ times $p$ matrix containing the levels $(p)$ of process parameters (and their interactions possibly, $n$ ) and $\varepsilon$ is a vector of independent random errors with mean 0 and variance $\sigma^{2}$. The prediction $y(\mathbf{x})$ at a point $\mathbf{x}$ is: $y(\mathbf{x})=\mathbf{x}$ est $(\boldsymbol{\beta})$, with est $(\boldsymbol{\beta})=\left(\mathbf{X}^{\mathrm{T}} \mathbf{X}\right)^{-1} \mathbf{X}^{\mathrm{T}} y$ : the least-squares estimator for $\beta$ based on the data. The prediction variance at $x$ (i.e., the variance of $y(\mathbf{x}))$ equals: $V(\mathbf{x})=\sigma^{2} \mathbf{x}\left(\mathbf{X}^{\mathrm{T}} \mathbf{X}\right)^{-1} \mathbf{x}^{\mathrm{T}}$ The standard I-optimal designs choose $\mathbf{X}$ (i.e., the settings of the experiments) to minimize the average prediction variance over an experimental region $(\mathbf{R})$ of interest, that is, they minimize:

$\mathrm{I}(\mathbf{X})=\int_{\mathbf{x} \in \mathbf{R}} \mathbf{x}\left(\mathbf{X}^{\mathrm{T}} \mathbf{X}\right)^{-1} \mathbf{x}^{\mathrm{T}} \mathrm{d} \mathbf{x} \cong \Sigma_{\mathbf{x} \in \mathrm{G}(\mathbf{R})} \mathbf{x}\left(\mathbf{X}^{\mathrm{T}} \mathbf{X}\right)^{-1} \mathbf{x}^{\mathrm{T}}$,

where $G(\mathbf{R})$ is a fine grid, covering the complete region of $\mathbf{R}$. If prior knowledge is available then low prediction variance may be desired especially in a subregion of $\mathbf{R}$ (i.e., the subregion where meeting of acceptance criteria is considered most likely). In such cases, (1) may be replaced by

$\operatorname{Iw}(\mathbf{X})=\Sigma_{\mathbf{x} \in \mathrm{G}(\mathbf{R})} \mathrm{w}(\mathbf{x}) \mathbf{x}\left(\mathbf{X}^{\mathrm{T}} \mathbf{X}\right)^{-1} \mathbf{x}^{\mathrm{T}}$,

where $w(\mathbf{x})$ is a pre-specified weight-function with weights that are different in the subregion compared to the rest of $\mathrm{G}(\mathbf{R})$. The criterion in (2) may be referred to as the weighted I- optimality criterion. In cases where multiple quality parameters need to be considered $\left(y_{1}, y_{2}, . ., y_{N}\right)$, this approach is also valid if it is possible to use the same independent variables and interactions for each response variable. It is important to note that the weight-function is user-defined. This is also the great advantage of this approach over standard, non-weighted, criteria, The user can put more weight to design points that correspond to settings of the input and process parameters that (based on previous experiments) are more likely to result in a final product meeting all requirements. Typically, the designs that are optimal with respect to the weighted $I$-criterion (Iw) cover the whole experimental region, but a relatively larger proportion of the design points will be located in and around the region where acceptance of the final product is deemed most likely. The approach therefore supports the improvements mentioned in the introduction: (a) using prior knowledge the design is focused on the optimal region and (b) the use of an optimality criterion ensures a lean design which can dramatically reduce the number of experiments in a standard design.

\section{Application of the Adapted I-Optimality Criterion in Process Design}

In order to establish such an approach, the abovementioned theoretical building blocks are combined into a practical workflow which consists of eight steps (Fig. 1). The first step is to collect historical and expert knowledge to define and prioritize all process parameters, product quality parameters, and their interactions (step 2). Based on this information, in step 3 a screening experiment (usually 2level) is performed to establish the relations between the highest priority process and product parameters. In step 4, regression models are defined on basis of the screening design. The purpose of these models is to predict the combinations of parameters and settings that lead to the desired product: the "preliminary design space". Step 5 defines the range of the experimental region for the optimization experiments, based on expert knowledge. Ideally, this region (partly) overlaps the initial region which means that the predictions in the next step are based on interpolation rather than extrapolation. The region is defined by a discrete grid where each point is a candidate setting (see Eqs. 1 and 2). It is clear that the grid points should reflect relevant and practically feasible settings. In step 6 , the prediction models are used to determine the preliminary design space. This means that for all points in the optimization region, the product quality parameters are predicted. The preliminary design space consists of those points for which the product quality parameters meet all quality requirements. Step 7 consists of attributing weights to the points inside and outside of the preliminary design space. The weights within the design space are usually set 


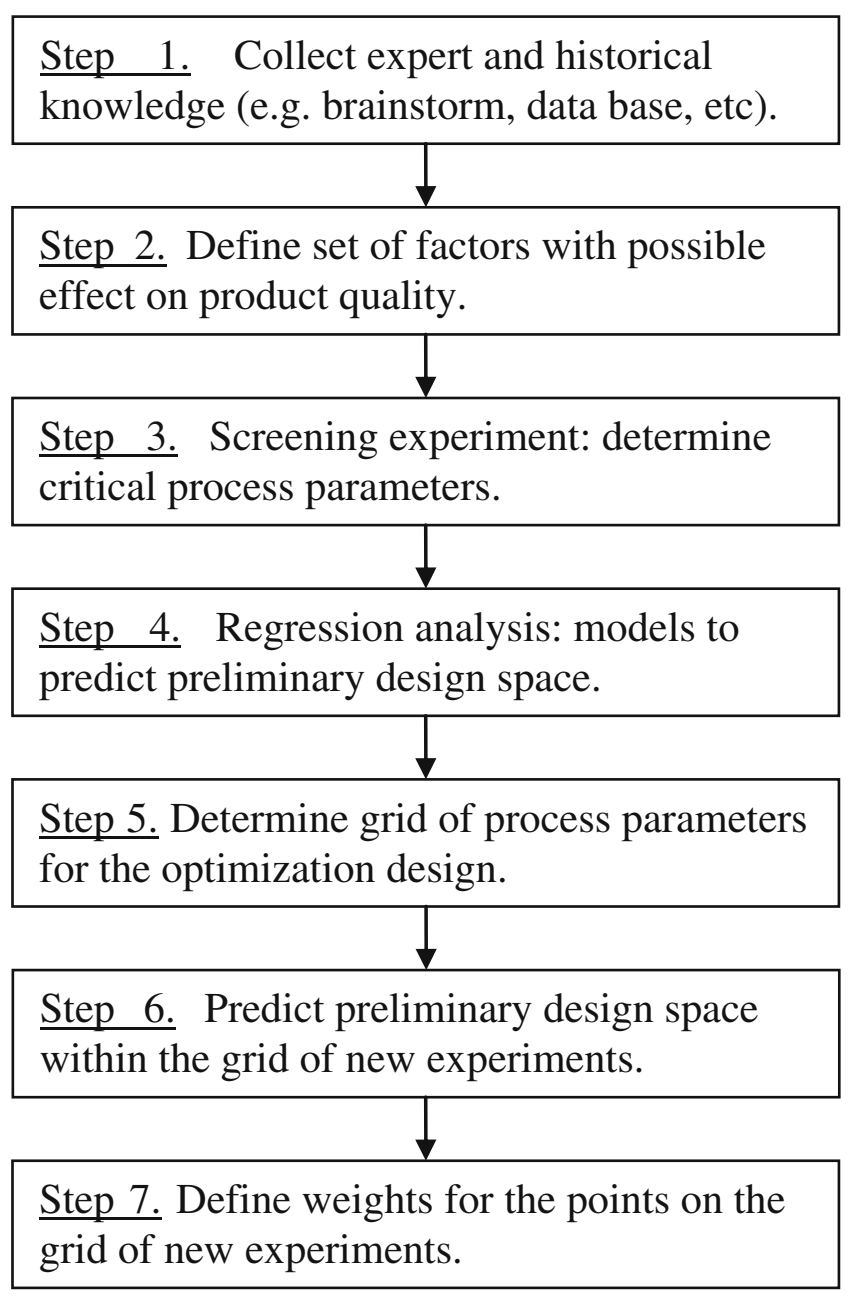

Step 8. Determine optimal number of runs (Iw criterion) and compare with alternative, standard design.

Fig. 1 Framework for an optimization strategy using multi-stage designs (high flexibility) and the $\mathrm{I}_{\mathrm{w}}$ optimality criterion (high efficiency)

to 1 . The weights outside this space are defined relative to the weights within the design space and express the confidence in the preliminary design space. Unfortunately, there is no objective criterion to define these weights. For this reason, expert opinion or a rule of thumb is used to define these weights. As a rule of thumb, we suggest to first calculate the fraction $(F)$ of the number of points inside the preliminary control space. Second, the weights outside of this region should be approximately $F /(2-2 F)$. This means that average weight inside of the preliminary design space $(1 \times \mathrm{F})$ is about twice as high as the weight outside of this region which again reflects the confidence of the user on the preliminary design space. In the analysis of the case study and the discussion, the aspect of weight selection is discussed in more detail. In step 8 , an exchange algorithm (3) is used to find the Iw optimal design. First, a desired number of design points is selected at random from a candidate set to form a starting design for the algorithm. In subsequent steps of the algorithm, exchanges of points in the design and the candidate set are considered and the exchange that results in the largest decrease of the Iw criterion is selected at each step. This is repeated until none of the possible exchanges yields a lower Iw criterion. During this procedure, it is constantly assured that all the candidate sets are able to assess all required effects by only considering sets which rank is larger than zero. The procedure is performed for several starting designs and the design with smallest value for the Iw criterion is chosen as the optimal design.

\section{Software}

The regression models have been calculated using SAS 9.1.3 (service pack 4, Copyright 2002-2003 by SAS institute Inc., Cary, NC, USA) for windows. All other calculates have been performed using home-made Matlab 7.7.0 software for windows (The Mathworks, MA, USA).

\section{Results}

In order to test the suggested approach, it was applied on a real-world industrial application: the process development of a tablet product. Before applying the proposed strategy of this paper, the process development had already been performed completely using standard experimental designs in two consecutive stages. However, in contrast to this study, no thorough framework had been used to connect these stages. Based on the existing data of both stages 1 and 2 , this section shows how to use the experiments of stage 1 to model a preliminary design space, how to use the Iw criterion to define the suggested optimal design (stage 2) and the comparison of the optimal design with the standard design that was used in practice. It is important to note that the Iw criterion was only used to define the optimization design (stage 2) and not the stage 1 screening design because these results are needed to define the Iw.. Because of the mathematical background of this approach, the number of experiments in the design and the optimality criterion can be used as objective criteria to compare the quality of the alternative and the original designs.

\section{Example Case: Pharmaceutical Process Development}

The manufacturing process of this tablet formulation consists of six unit operations: granulation, drying and 
milling, final mixing with lubricant, compression into tablets, and film coating. Before constructing the Iw, as a very first step in the process development, a brainstorm meeting was performed with several in-house experts to determine the list of 108 parameters and their estimated influence on the 12 relevant product attributes. From this inventory, the expert scores were summed leading to a selection of ten allegedly important parameters that is further investigated in a two-level screening design (i.e., investigating a "high" and a "low"-level) with respect to two important product attributes: mean crushing strength and mean disintegration time of tablets. The process parameters are: amount of granulation water, addition time of water during granulation, speed mixer, granulation time (from the unit of granulation); loss on drying (from the unit of drying); mixing speed blender, blending time (from the unit of blending); tabletting speed, pre-compression force, main compression force (from the unit operation of compression). Based on the screening experiment three process parameters were identified and the minimum and maximum of the levels were defined. Two of these were adjusted (amount of granulation time and addition time of water) because it was observed that the preliminary design space was at the edge of the investigated range while it is better to have this region at the center of the investigated range. Finally, a 32-run three-level full factorial experiment was performed to find the optimal settings of the three process parameters identified in the screening experiment. The reader is referred to Table 1 for the levels of the screening and the original follow-up design.

The alternative Iw-criterion based approach to connect stages 1 and 2 and to determine the optimal stage 2 experiments was performed as follows. First, the results from the screening design were used to find a quantitative model for the two response parameters, mean crushing strength $\left(y_{1}\right)$ and mean disintegration time $\left(y_{2}\right)$ based on the three most important process parameters: amount of granulation water $\left(x_{1}\right)$, addition time of granulation water $\left(x_{2}\right)$ and granulation time $\left(\mathrm{x}_{3}\right)$ :

$$
\begin{aligned}
y_{1} & =1.57-0.29 x_{1}+0.13 x_{2}-0.18 x_{3}+0.19 x_{1} x_{2} \\
& +0.06 x_{2} x_{3}-0.02 x_{1} x_{3} \\
\text { - } y_{2} & =0.79-0.02 x_{1}-0.04 x_{2}-0.07 x_{3}-0.53 x_{1} x_{2} \\
& -0.49 x_{2} x_{3}+0.56 x_{1} x_{3} .
\end{aligned}
$$

Next, a grid is defined that corresponds to the range of product parameters that should be investigated in the optimization experiments. For each point on the grid (i.e., for each possible setting of the product parameters in the investigated range), the response parameters are predicted using the abovementioned models. The combination of grid points for which the predicted response parameters

\begin{tabular}{|c|c|c|}
\hline & \multicolumn{2}{|l|}{ Levels } \\
\hline & $\begin{array}{l}\text { Screening } \\
\text { experiment } \\
\text { (stage 1) }\end{array}$ & $\begin{array}{l}\text { Follow-up } \\
\text { experiment } \\
\text { (stage 2) }\end{array}$ \\
\hline \multirow[t]{3}{*}{ Granulation time (min) } & 4 & 4 \\
\hline & 6 & 5 \\
\hline & & 6 \\
\hline \multirow[t]{3}{*}{ Amount of granulation water $(\mathrm{g})$} & 6000 & 5500 \\
\hline & 7500 & 6250 \\
\hline & & 7000 \\
\hline \multirow[t]{3}{*}{ Addition time of water (s) } & 30 & 60 \\
\hline & 120 & 90 \\
\hline & & 120 \\
\hline
\end{tabular}
meet the quality requirements is considered the prelimi-
Table 1 Levels for factors in screening (32 runs) and follow-up stage (32 runs)

nary design space. In this study, these requirements are mean crushing strength $>90 \mathrm{~N}$ and mean disintegration time between 1 and $15 \mathrm{~min}$. Note that it is important to realize that if the range of the screening design and the optimization designs are not equal, the prediction of the response parameters relies on extrapolation of the derived models. Although this situation is not desired for prediction purposes, it is a valid approach to derive a first estimate. Grid points in the preliminary design space (i.e., the predictions satisfy all requirements) receive a weight equal to 1 , whereas points with at least one constraint not satisfied obtain a smaller weight. The weight given to the points outside of the preliminary design space should reflect the certainty of the results of earlier experiments.

Based on these settings, finding the optimal design is a matter of automatically evaluating different combinations of candidate design points, as is described in step 8 of Fig. 1. Because a higher weight is given to points in the preliminary design space, this space has a high positive influence on the final experimental settings that are selected. However, it is crucial that the points outside of this region are also considered as candidates although with a lower importance.

A special point of attention is the selection of the values of the weights which can reflect the percentage of the points in the grid that are in the preliminary design space. In the case study, only a small fraction of $9.1 \%$ grid points lie in the preliminary design space. This should be taken into account when choosing the weights. For weights 0.1 ( $\sim 91 \%$ of the grid points) and 1.0 ( $\sim 9 \%$ of the grid points), the average I-criterion approximately is the same inside and outside of the preliminary design space. By choosing $w=$ 0.05 we make the average prediction variance inside the control space two times as important. Because there is no 
objective criterion to set these weights, in this case, the sensitivity of the total approach was investigated by using different weights $(0.025,0.05,0.1,0.3$, and 0.5$)$ and calculating their corresponding optimal designs (Table 2).

Table 2 shows that the lower the weight of the points outside the preliminary design space, the less design points are required to find a design with the same or better characteristics than the full factorial design. This is because more design points will stem from the preliminary design space. It is also clear that with a weight of 0.1 (i.e., the average weight outside and inside of the preliminary design space is equal), this approach still finds a design with less required experiments than the full factorial design (i.e., 27 instead of 32, a reduction of $15 \%$ ). For the weights of 0.05 and 0.025 , the reductions are $21 \%$ and $31 \%$, respectively. For hypothetical cases where the preliminary design space is given less average importance than the other space, the results converge to 32-run designs which are equal to the full factorial design. This means that the concept of optimality criteria will lead to no extension of the number of experiments compared to standard designs: at the worst the number of experiments is equal. Table 3 compares the settings for the three process parameters for both the optimal design (weight of 0.05 ) and the original design. It is clearly seen that the new design contains practical levels that can easily be set (e.g., integers or values with limited decimals) and is comparably practical to the original design. This is ensured by a proper definition of the grid points. Finally, the improved quality of this approach has become clear from the lower number of experiments, lower

Table 2 The Iw values for different weights and different number of experiments in the experimental design

\begin{tabular}{llllll}
\hline & $w=0.025$ & $w=0.05$ & $w=0.1$ & $w=0.3$ & $w=0.5$ \\
\hline FFD (N=32) & $\mathbf{0 . 0 2 4 7}$ & $\mathbf{0 . 0 2 8 1}$ & $\mathbf{0 . 0 3 8 4}$ & $\mathbf{0 . 0 7 2 3}$ & $\mathbf{0 . 1 0 6 3}$ \\
$N=20$ & 0.0271 & 0.0358 & & & \\
$N=21$ & 0.0257 & 0.0340 & & & \\
$N=22$ & $\underline{0.0243}$ & 0.0320 & & & \\
$N=23$ & 0.0233 & 0.0303 & & & \\
$N=24$ & & 0.0289 & 0.0430 & & \\
$N=25$ & & $\underline{0.0279}$ & 0.0414 & & \\
$N=26$ & & 0.0267 & 0.0399 & & \\
$N=27$ & & 0.0256 & $\underline{0.0380}$ & & \\
$N=28$ & & 0.0248 & 0.0369 & 0.0772 & 0.1160 \\
$N=32$ & & & 0.0320 & $\underline{0.0669}$ & $\underline{0.1004}$ \\
\hline
\end{tabular}

The Full Factorial Design (FFD) is considered as a reference (bold) The Iw value that is smaller than the reference with the least number of experiments is underlined

The blank cells represent trivial results which do not contribute to this study
Iw-criterion and the framework with a higher focus on the preliminary design space.

\section{Discussions}

In the presented strategy, there are two main aspects that need special attention. The first one relates to the goal of process development: finding optimal settings or the design space, while the second one relates to the methodology presented in this paper: determining the weights.

Irrespective of the approach followed (using the Iw criterion or using standard sequential designs without a systematic framework), the key assumption is that the screening design and the correspondingly derived preliminary design space are a good prediction of the final design space. This is not necessarily the case, even though these are the best available estimates. This risk becomes smaller if the available knowledge and level of expertise is higher which in its turn should lead to a better screening design (i.e., containing the proper factors and levels). In addition, a more efficient design approach, as shown in this study, also opens the opportunity to use the same number of experiments compared to standard approaches. The excess of experiments can be used to focus on areas where prior knowledge or expert opinion is less mature, lowering the unavoidable subjective part of process development. Moreover, the advantage of the Iw criterion is that there is an explicit focus for the optimization design on the region outside of the preliminary design space. In the case where the preliminary design space is shifted from the true (but partly unknown) one, it is likely that the latter is still included in the optimization design. An alternative approach might be to not only set higher weights (e.g., 1) to the grid points in the preliminary design space but also higher weights (e.g., 2) to the grid points $20 \%$ around the borders of the preliminary design space. The points outside both of these regions are given a lower weight (e.g., 0.05).

The second point of attention corresponds to selecting the values of the weights. Essentially, this comes down to the level of confidence the researchers have with respect to the existing knowledge, the screening design, and the preliminary design space. This is not different from the standard approach although it can be difficult to translate a subjective feeling into a numeric value. For this reason we have suggested a rule of thumb which ensures equal average weight values inside and outside of the preliminary design space. Moreover, this paper also shows what happens if different values are selected: the results can vary considerably. It might be a good approach to calculate different options (using different set of weights) and 
Table 3 The settings of the three process parameters for both the Iw-based optimal design and the original full factorial design with replicated center points

\begin{tabular}{|c|c|c|c|c|c|c|}
\hline \multirow[t]{2}{*}{ Run number } & \multicolumn{3}{|l|}{ Optimal design } & \multicolumn{3}{|l|}{ Original design } \\
\hline & $\begin{array}{l}\text { Amount of } \\
\text { granulation water }(\mathrm{g})\end{array}$ & $\begin{array}{l}\text { Addition time } \\
\text { of water (s) }\end{array}$ & $\begin{array}{l}\text { Granulation } \\
\text { time (min) }\end{array}$ & $\begin{array}{l}\text { Amount of } \\
\text { granulation water }(\mathrm{g})\end{array}$ & $\begin{array}{l}\text { Addition time } \\
\text { of water (s) }\end{array}$ & $\begin{array}{l}\text { Granulation } \\
\text { time (min) }\end{array}$ \\
\hline 1 & 5675 & 78 & 5 & 5500 & 120 & 6 \\
\hline 2 & 5000 & 78 & 4.8 & 6250 & 90 & 5 \\
\hline 3 & 6500 & 102 & 5 & 6250 & 90 & 5 \\
\hline 4 & 5750 & 96 & 5 & 6250 & 60 & 5 \\
\hline 5 & 5000 & 99 & 4 & 6250 & 60 & 6 \\
\hline 6 & 5600 & 99 & 4 & 5500 & 60 & 6 \\
\hline 7 & 5600 & 120 & 4 & 5500 & 60 & 5 \\
\hline 8 & 5000 & 120 & 4 & 5500 & 60 & 4 \\
\hline 9 & 5750 & 99 & 5 & 7000 & 60 & 5 \\
\hline 10 & 5000 & 99 & 4.8 & 7000 & 90 & 4 \\
\hline 11 & 5750 & 96 & 5 & 6250 & 120 & 6 \\
\hline 12 & 5600 & 99 & 4 & 6250 & 90 & 5 \\
\hline 13 & 6500 & 78 & 6 & 5500 & 90 & 4 \\
\hline 14 & 5675 & 78 & 4 & 7000 & 120 & 5 \\
\hline 15 & 6500 & 120 & 4 & 7000 & 60 & 4 \\
\hline 16 & 5000 & 99 & 4 & 6250 & 90 & 5 \\
\hline 17 & 5600 & 120 & 6 & 6250 & 120 & 4 \\
\hline 18 & 6500 & 120 & 6 & 5500 & 90 & 5 \\
\hline 19 & 5000 & 102 & 6 & 6250 & 120 & 5 \\
\hline 20 & 5000 & 78 & 6 & 7000 & 60 & 6 \\
\hline 21 & 5000 & 120 & 5 & 6250 & 90 & 5 \\
\hline 22 & 5000 & 78 & 4 & 6250 & 60 & 4 \\
\hline 23 & 5000 & 78 & 4 & 5500 & 90 & 6 \\
\hline 24 & 6500 & 78 & 4 & 6250 & 90 & 5 \\
\hline 25 & 5000 & 102 & 4.8 & 6250 & 90 & 6 \\
\hline 26 & & & & 7000 & 90 & 5 \\
\hline 27 & & & & 7000 & 120 & 4 \\
\hline 28 & & & & 5500 & 120 & 5 \\
\hline 29 & & & & 5500 & 120 & 4 \\
\hline 30 & & & & 6250 & 90 & 4 \\
\hline 31 & & & & 7000 & 90 & 6 \\
\hline 32 & & & & 7000 & 120 & 6 \\
\hline
\end{tabular}

visually compare the results to a standard optimization design. From this the researcher can learn what design (i.e., weight) corresponds to the available but subjective level of confidence.

\section{Conclusions}

This paper presents a framework in which experiments can be designed for process development. Unlike standard approaches, prior knowledge (from historical processing data, expert knowledge, or a screening design) is explicitly used to set up an optimization design, focused on process parameter settings which are most likely to be highly important. With this approach it becomes possible to construct dedicated experimental designs that perform equally or better than standard designs but require fewer experiments. A crucial aspect in this framework is the novel Iw optimality criterion for finding optimal designs while taking into account parameter sub-regions which seem to be more important without neglecting other regions to avoid missing important information. 
The developed strategy was applied on a pharmaceutical case study from which both a screening and an optimization design have been developed in a traditional manner. In contrast, this study shows from a theoretical perspective that a reduction of $21 \%$ (seven experiments) is possible while the theoretical power or the performance of the approach was not affected compared to the traditional design. In general, the reduction of experiments depends on the problem at hand but in the worst case, the proposed $\mathrm{I}_{\mathrm{w}}$ strategy will converge to the standard design. Moreover, the development of such a tailor-made optimization strategy will not take more resources than the traditional approach because the calculations are performed automatically. This also allows the use of this approach in parallel to a standard approach, as an objective critical reviewer.

\section{References}

1. International Conference of Harmonisation (2008) ICH Harmonised Tripartite Guideline: Q8(R2) Pharmaceutical Development (www.ich.org/LOB/media/MEDIA4986.pdf)

2. Lewis GA, Mathieu D, Phan-Tan-Luu R. Pharmaceutical experimental design. New York: M. Dekker; 1999.

3. Armstrong NA. Pharmaceutical experimental design and interpretation. 2nd ed. London: Taylor \& Francis; 2006.

4. Lunney PD, Cogdill RP, Drennen III JK. Innovation in pharmaceutical experimentation Part 1: review of experimental designs used in industrial pharmaceutics research and introduction to Bayesian D-optimal experimental design. J Pharm Innov. 2008;3:188-203.

5. Atkinson AC, Donev AN, Tobias RD. Optimum experimental designs, with SAS. New York: Oxford University Press; 2007.

6. Boyd S, VandenBerghe L. Convex optimization. Cambridge UK: Cambridge University Press; 2004. 